\title{
An Efficient Preprocessing Technique for Noise Reduction in Ear Verification System
}

\author{
Sude Tavassoli \\ Department of Computer Engineering, \\ Islamic Azad University, Roudsar branch, Iran
}

\author{
Mahboubeh Yaqubi \\ Department of Computer Engineering, \\ Islamic Azad University, Lahijan branch, Iran
}

\begin{abstract}
Today, Biometric systems are considered superior in technological developments, because they provide a nontransferable means of identifying people not just cards or badges. The image enhancement step is designed to reduce noise in this area. The key point about an identification method that is "nontransferable" means it cannot be given or lent to another individual so nobody can get around the system they personally have to go through the control point. The image enhancement before feature extraction system can be very efficient. In this paper a new method is proposed to raise the performance of an ear verification system, since at first, using hybrid denoising method, the noises removed from ear image and then the next step denoisy image is used for verification system. Experimental results in this study show that Gaussian noises well removed from the ear images and has acceptable affect on verification accuracy.
\end{abstract}

\section{Keywords}

Image denoising, Preprocessing, Verification system, Adaptive Neuro-Fuzzy Inference System, Fuzzy Wavelet Shrinkage.

\section{INTRODUCTION}

Biometrics are automated methods of recognizing an individual based on their physiological (fingerprints, face, ear, iris) $[1,2,3]$ or behavioral characteristics (gait, signature) [4]. Each biometric has its strengths and weaknesses and the choice typically depends on the application [5]. Biometric systems work by first capturing a sample of the feature, such as taking a digital image. After the segmentation process [6], the sample is then transformed using some mathematical function into a biometric template. The biometric template will provide a normalized, efficient and high discriminating representation of the feature, which can then be objectively compared with other templates in order to determine the identity $[7,8,9]$. So that image denoising is one of the important preprocessing of verification systems. Performance of feature extraction methods and verification systems depend on quality of input image $[10,11]$.

HMAX is a feature extraction method and this method is motivated by a quantitative model of visual cortex. In its simplest version, the standard model consists of four layers of computational units where simple $\mathrm{S}$ units, which combine their inputs with Gaussian-like tuning to increase object selectivity, alternate with complex $\mathrm{C}$ units, which pool their inputs through a maximum operation, thereby introducing gradual invariance to scale and translation. The model has been able to quantitatively duplicate the generalization properties exhibited by neurons in infer temporal monkey cortex (the so-called view-tuned units) that remain highly selective for particular objects (a face, a hand, a toilet brush) while being invariant to ranges of scales and positions. Also, SVMs are classifiers which have demonstrated high generalization capabilities in many different tasks, including the object recognition problem. In this reference, they combined these two techniques for the robust Ear verification problem. In addition to, demonstrated that this method is rotate and scale-invariant, and also, in experiment, it was found that, using of Gaussian filter in HMAX model in compared to using of Gabor filter, increases performance of ear recognition [2, 3, 11].

Noise can be occurred in digital images during acquisition and/or transmission images [12]. Several methods have been proposed for removal noises from digital images. Most of them used Median Filter (MF) methods and other versions of median filter, but MF only have a good ability to noise reduction in low noise ratio. Besides in most of the times especially in high noise ratio, MF result in destroyed vital textures and edges $[13,14,15]$. Recently, researchers focused on two steps of noise detection and noise replacement methods [16]. Additionally not only noise removal process utilize as a simple preprocess in few application but also today has been using in different applications. Previously, several image denoising techniques have been used successfully in many image processing applications; in [17] authors proposed two models based on loss and penalty minimization on the face image. Mean shift algorithm presented to remove noises in $3 \mathrm{D}$ images which generated by estimation methods [18]. A new fuzzy filter based on interval-valued fuzzy sets for image filtering discussed by Bigand and Colot [19]. In application of multi spectral images an another new wavelet shrinkage algorithm based on fuzzy logic denoising method suggested in [20] and experimented on single and multi-channel images. Moreover, there are other applications of noise removal in new various types of images reported for pinned-type CMOS image sensors [21], Color images [22], video images [23], and real time image on chip [24].

The verification process is based on whether a person is who he/she claims to be $[7,9]$. These cases, the relationship between denoising and preservation of important details are very essential and require a proper algorithm for denoising. By considering the noise uncertainty in image pixels, fuzzy logic based methods $[12,14,15,20,25,26]$ also have been increasingly proposed which often perform in three phases: image fuzzification, modification of membership values and image deffuzification. In fact, the image data transfers from the input level into the membership level and then membership values can be modified with fuzzy rule based methods. In our proposed method, at first phase, Adaptive Neuro-Fuzzy 
Interface System (ANFIS) detects noisy pixels in ear image and then, Fuzzy Wavelet Shrinkage (FWS) will change only noisy pixels. At next phase, denoised images use for verification system. The main target of this paper is enhancement of verification system using efficient noise reduction from ear images with preserve quality. This proposed method in the first phase increases the accuracy and preserve normal texture of image, then in the second phase, improves verification system. The paper is organized as follows: our previous proposed hybrid method, ANFIS-FWS is described section 2. Section 3 demonstrates the proposed method in this paper for verification system enhancement. in section 4 experimental results of proposed method are evaluated in gray scale test images and ear images, Finally the conclusions and suggestions for future works are shown in section 5 .

\section{ANFIS-FWS DENOISING METHOD}

As norm in literature, ear verification systems have been presented in three main steps of Preprocessing, Feature extraction, and Classification [7] as has shown in figure 1. The focus of contribution in this paper is considered on preprocessing phase by denoising from the ear images.

A hybrid two-step method has been proposed for noise reduction from digital images, which part of it previously also has been used by these authors [14]. The proposed method at the first phase, ANFIS [12] identifies Noisy pixels with high accuracy, then FWS [27] is used based on the information of noisy and uncorrupted noisy pixels, so noisy pixel is replaced by new some value and unnoisy pixel remain naturally [28]. The main structure of proposed method for denoising is presented as figure 2. At the first stage, to noise detection, a training image with small size without any particular is used for learning, then this trained ANFIS is used for noise detection, this section of the paper already is used and described in $[12,14]$.

\subsection{Anfis}

Because of uncertainty of noise and preserving important details, to detection of noisy pixels using Adaptive Neuro-Fuzzy Inference System (ANFIS) is suggested for high accuracy. ANFIS is a fuzzy inference system implemented in the framework of adaptive networks. ANFIS serves as a basis for constructing a set of fuzzy if-then-else rules with appropriate membership functions to generate the simulated input-output pairs. The main structure of an ANFIS [12] also shown in figure 3 . At the first stage, to noise detection, a training image with small size without any particular is used for learning, then this trained ANFIS is used for noise detection, this section of the paper already is used and described in [14,27].

\subsection{Fws}

In this method, a proper Fuzzy norm is used instead of the shrinkage factor [14]. As if all two neighboring around a wavelet coefficient ws, $d$ in a location of $(i, j)$ is contained a large (small) coefficient, then can be a sign for the proper signal (noise) is there. As a principle that if a coefficient is small and neighbor coefficients are big then can be assign to neighbors more importance.

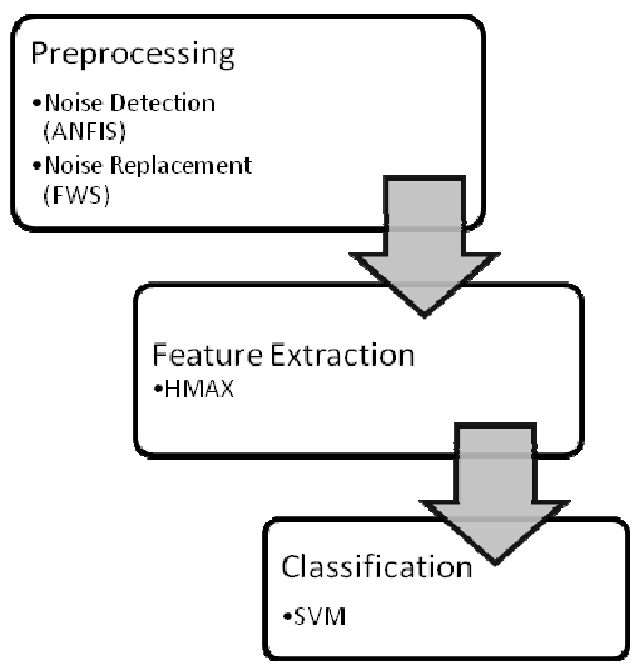

Figure 1. Our ear verification system [7]

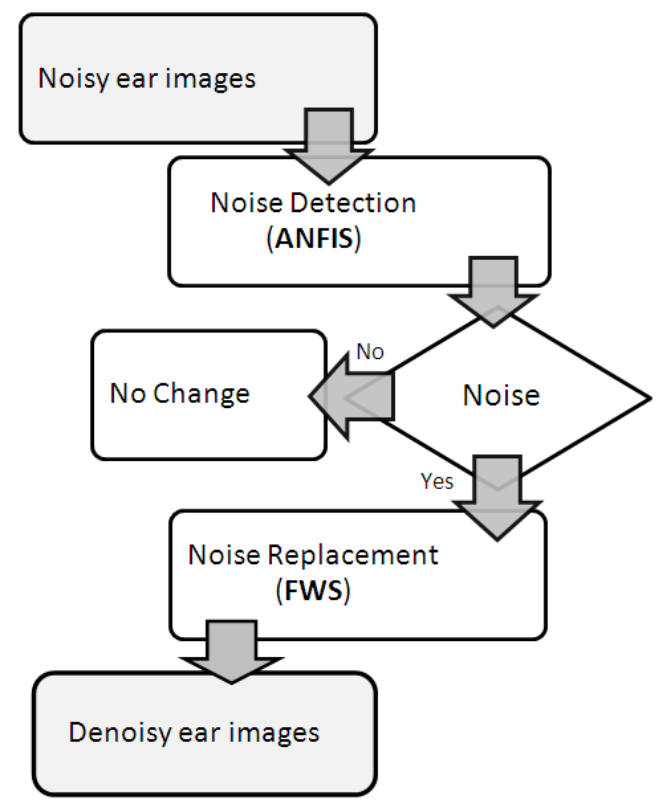

Figure 2. Proposed ANFIS-FWS for denoising [14]

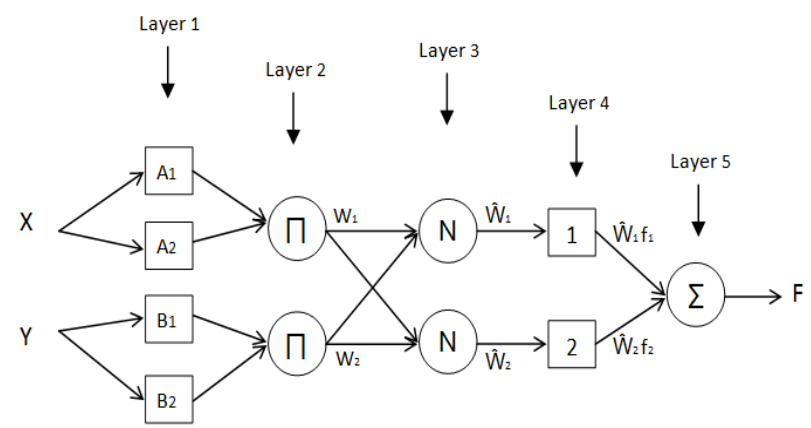

Figure 3. The structure of ANFIS [12] 
Therefore can defined a fuzzy rule, like Rule 1 that the variable $\mathrm{X}$ represents average of wavelet coefficients in the $(2 \mathrm{k}+1)$ neighborhood around a place (i, j) and relationship of neighborhood contains large and small coefficients.

\section{Fuzzy Rule1:}

IF (|xs,d (i,j)|) is a large variable AND (|ws,d $(i, j) \mid)$ is a large coefficient) OR (|xs,d $(i, j) \mid$ is a large variable THEN (ws,d $(i, j))$ is a proper signal. If the membership degree has value 1 , coefficient is a proper signal certainly and should not be changed (shouldn't be shrink), while a degree zero indicates that the coefficient is noise certainly and should be set equal to zero (should be shrink) [14].

\section{PROPOSED APPROACH}

The preprocessing step is one of the essential steps in biometric verification systems [7]. In this study, a denoising method for ear images presents to enhance verification performance. Firstly, using ANFIS and FWS that introduced in section 2, the noise on the ear image is removed and then at the next phase, enhanced image are used for verification system. Then, we use of HMAX model to extract features from ear images. In this method, it is introduced a novel set of features for robust ear recognition. This method investigates the generalization power of $\mathrm{C} 2$ features, inspired by the hierarchical organization of the primate visual ventral stream, over four important pattern recognition problems. Our visual system achieves high efficiency by means of neurons responsive to complex features which are themselves built upon simple orientation features in the visual hierarchy [2, $3,11]$. The main structure of the proposed method is shown at figure 4.

\section{EXPERIMENTAL RESULTS}

In this section, first of all, the performance of proposed method is evaluated by some gray level test images as visual including Sail, Baboon, Peppers, and Lena in comparison with Median Filter (MF) [28], Iterative Median Filter (IMF) [29], Fuzzy Filter (FF) [30], and proposed method in figures of 5 to 8.

For biometric evaluation, we use a subset of USTB ear database [31]. Figure 3 shows three images manually extracted of one subject. This database includes 180 Ear images from 60, with 3 images per person. In the preprocessing step the ear images are cropped to a size of $80 \times 150$ pixels. The normalization step includes geometric normalization, masking and photometric normalization. In this phase all the images are scaled to a standard 150x150 size. Next all non-ear areas, like hair, background etc. are masked. Different levels of masking are experimented for finding the best one to get as good performance as possible for the algorithm. Finally the images are normalized for illumination. After, we used Gaussian noise with different standard deviation $\sigma=1 \%, 5 \%, 10 \%, 15 \%$, and $20 \%$ on all normalized ear images of Ear dataset is applied.

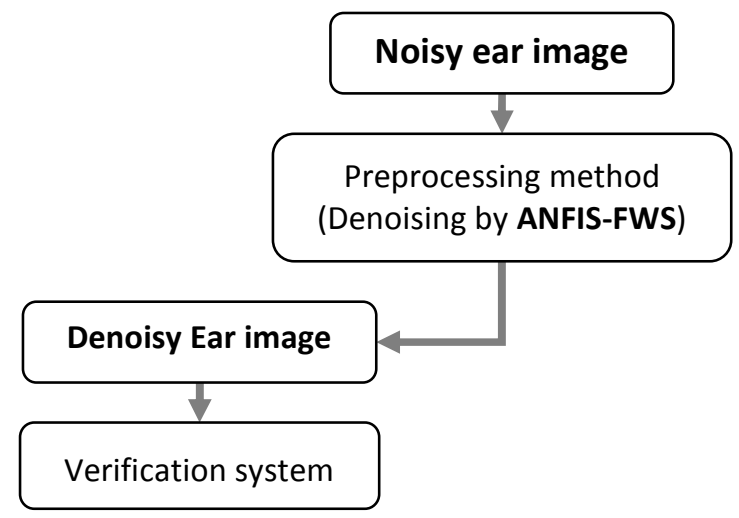

Figure 4. Main structure of Preprocessing proposed method

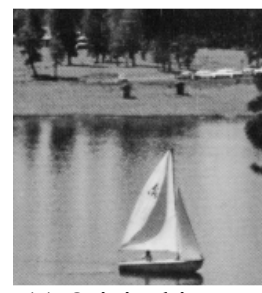

(a) Original image

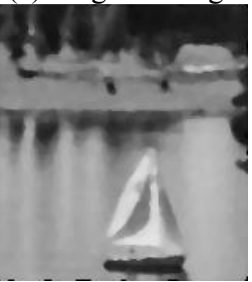

(d) IMF

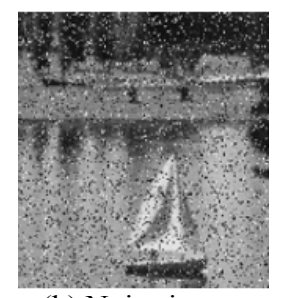

(b) Noisy image

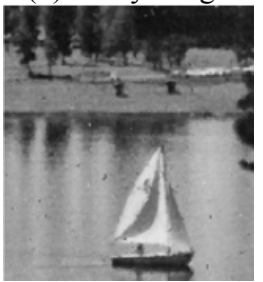

(e) FF

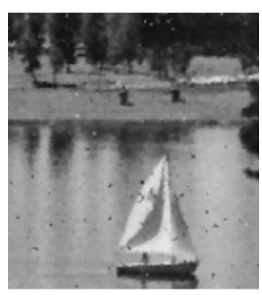

(c) $\mathrm{MF}$

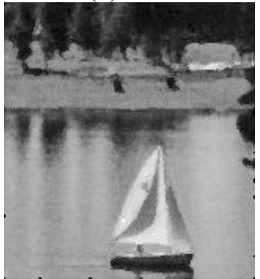

(f) Proposed method
Figure 5. Comparison of the restored images of methods for Lena by impulse noise $20 \%$

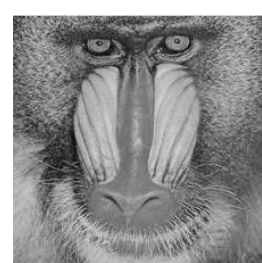

(a) Original image

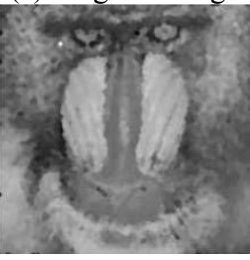

(d) IMF

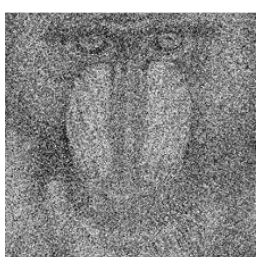

(b) Noisy image

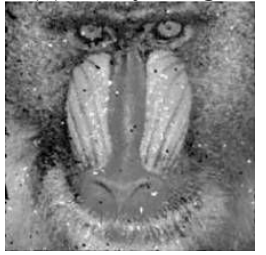

(e) $\mathrm{FF}$

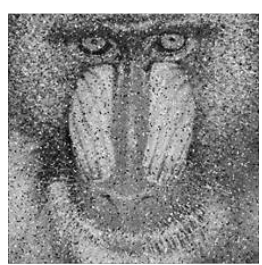

(c) MF

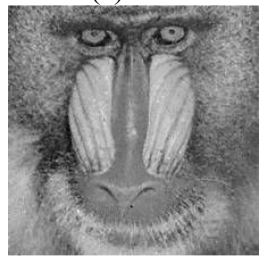

(f) Proposed method
Figure 6. Comparison of the restored images of methods for Baboon by impulse noise $40 \%$ 


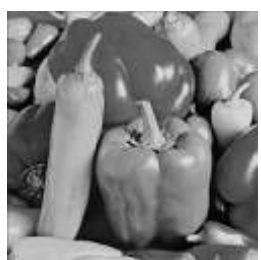

(a) Original image

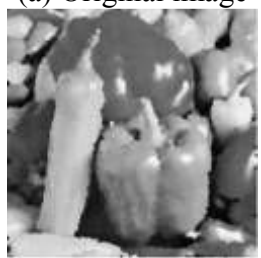

(d) IMF

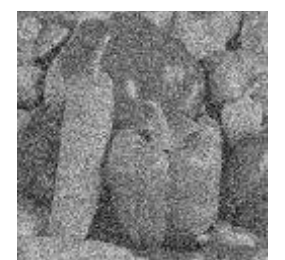

(b) Noisy image

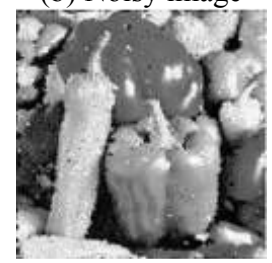

(e) FF

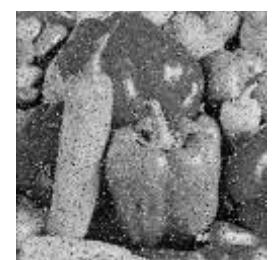

(c) $\mathrm{MF}$

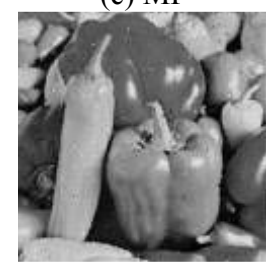

(f) Proposed method
Figure 7. Comparison of the restored images of methods for Peppers by impulse noise $50 \%$

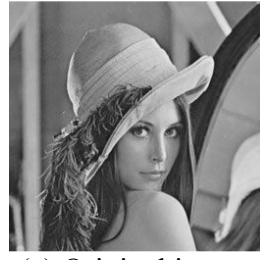

(a) Original image

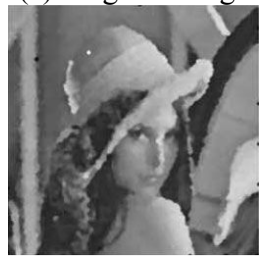

(d) IMF

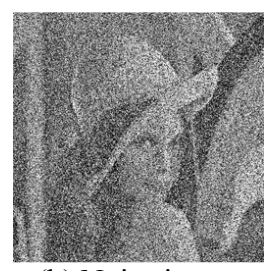

(b) Noisy image

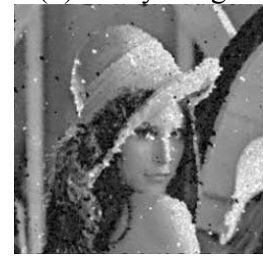

(e) FF

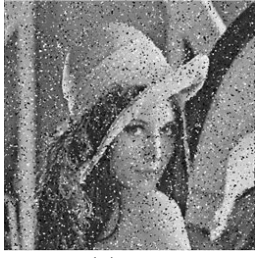

(c) MF

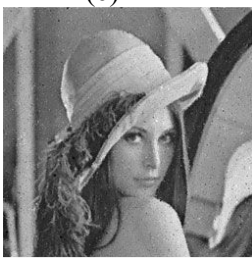

(f) Proposed method
Figure 8. Comparison of the restored images of methods for Lena by impulse noise $50 \%$

After applying the Gaussian noises, ANFIS-FWS denoising method [27] has performed on noisy images and then for evaluation the denoising method calculated objective quantitative, measure which is used for comparison that called Peak Signal-to-Noise Ratio (PSNR) criteria, as defined by equation (1) [14]:

$$
P S N R=10 \log _{10}\left(\frac{255^{2}}{M S E}\right)
$$

Also Mean Squared Error (MSE) obtained based on equation (2) [14]:

$$
M S E=\frac{\sum_{i=1}^{N} \sum_{j=1}^{M}\left(x_{i j}-u_{i j}\right)^{2}}{M \times N}
$$

As to the equation $M$ and $N$ are defined as dimensions of image, and $x_{i j}, u_{i j}$ indicates the pixel value in place $i, j$ for the first image (noisy) and enhanced image respectively. If MSE is a low value, denoising accuracy is high. In this part of experiments, the twodimensional correlation criterion (COR) is used to compare the proposed method with other methods also. The equation (3) defines the COR [14]:

$$
C O R=\frac{\sum_{i=1}^{N} \sum_{j=1}^{M}\left(x_{i j}-\bar{x}\right)\left(u_{i j}-\bar{u}\right)}{\sqrt{\left(\sum_{i=1}^{N} \sum_{j=1}^{M}\left(x_{i j}-\bar{x}\right)^{2}\right)\left(\sum_{i=1}^{N} \sum_{j=1}^{M}\left(u_{i j}-\bar{u}\right)^{2}\right)}}
$$

Where $x_{i j}$ is noisy image, $u_{i j}$ is enhanced image, $\bar{x}$ is mean value of noisy image, and $\bar{u}$ is mean value of enhanced image.

First, to evaluate our proposed method, the new hybrid of Adaptive Neuro Fuzzy inference system and Fuzzy wavelet Shrinkage (ANFIS-FWS) [14] has been compared with other common methods such as Median filter, Wiener filter, Fuzzy Wavelet Shrinkage (FWS) [32] on Ear noisy images based on the Mean Square Error (MSE) in table (1). In results can be seen ANFIS-FWS denoising method could removed noise with different standard deviation $\% 1, \% 5 . \% 10, \% 15$, and $\% 20$ on images, properly and with high accuracy. The proposed method has taken lowest MSE than other Methods.

Table 1. MSE results for denoising method and other methods

\begin{tabular}{cccccc}
\hline Method & $\mathbf{2 0} \%$ & $\mathbf{1 5} \%$ & $\mathbf{1 0} \%$ & $\mathbf{5 \%}$ & $\mathbf{1} \%$ \\
\hline \hline Noise & 1009.90 & 773.51 & 545.62 & 259.58 & 35.70 \\
Median & 450.74 & 335.18 & 230.48 & 107.70 & 20.09 \\
Wiener & 282.829 & 206.54 & 134.22 & 58.82 & 13.35 \\
FWS & 177.21 & 142.42 & 103.12 & 54.99 & 29.03 \\
ANFIS-FWS & 146.27 & 116.98 & 84.55 & 42.36 & 10.70
\end{tabular}

Similarly, in table 2 performance of proposed method and famous method are presented by PSNR.

Table 2. PSNR results for denoising method and other methods

\begin{tabular}{cccccc}
\hline Method & $\mathbf{2 0} \%$ & $\mathbf{1 5} \%$ & $\mathbf{1 0} \%$ & $\mathbf{5 \%}$ & $\mathbf{1 \%}$ \\
\hline \hline Noise & 18.09 & 19.25 & 20.76 & 23.99 & 32.60 \\
Median & 21.59 & 22.88 & 24.50 & 27.81 & 35.10 \\
Wiener & 23.62 & 24.98 & 26.85 & 30.44 & 36.88 \\
FWS & 25.65 & 26.60 & 28.00 & 30.73 & 33.50 \\
ANFIS-FWS & 26.48 & 27.45 & 28.86 & 31.86 & 37.84 \\
\hline
\end{tabular}

In Table 2 performance of proposed method and famous method are listed by COR. 
Table 3. COR results for denoising method and other methods

\begin{tabular}{cccccc}
\hline Method & $\mathbf{1} \%$ & $\mathbf{5 \%}$ & $\mathbf{1 0} \%$ & $\mathbf{1 5} \%$ & $\mathbf{2 0} \%$ \\
\hline Noise & 0.9984 & 0.9886 & 0.9761 & 0.9652 & 0.9546 \\
Median & 0.9991 & 0.9995 & 0.9899 & 0.9848 & 0.9799 \\
Wiener & 0.9995 & 0.9982 & 0.9957 & 0.9929 & 0.9901 \\
FWS & 0.0996 & 0.9991 & 0.9969 & 0.9956 & 0.9942 \\
ANFIS- & & & & & \\
FWS & 0.9997 & 0.9989 & 0.9983 & 0.9973 & 0.9963 \\
\hline
\end{tabular}

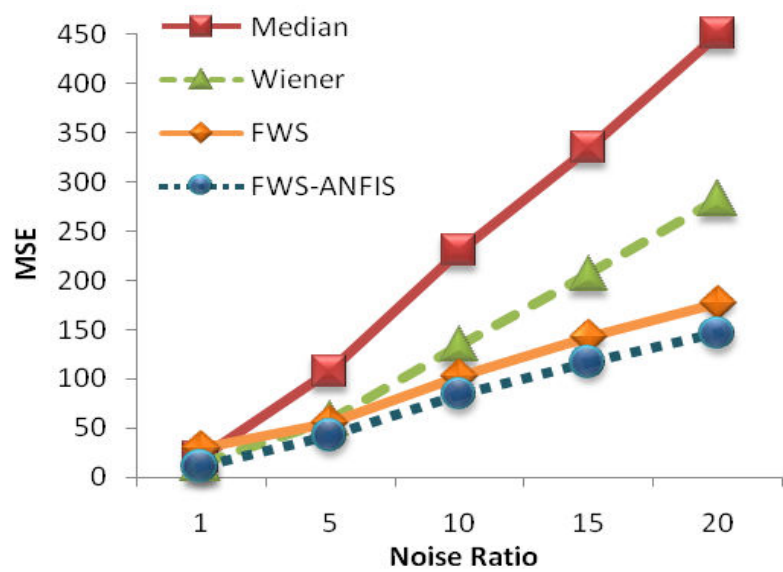

Figure 9. Comparison of proposed method performance and other methods by MSE

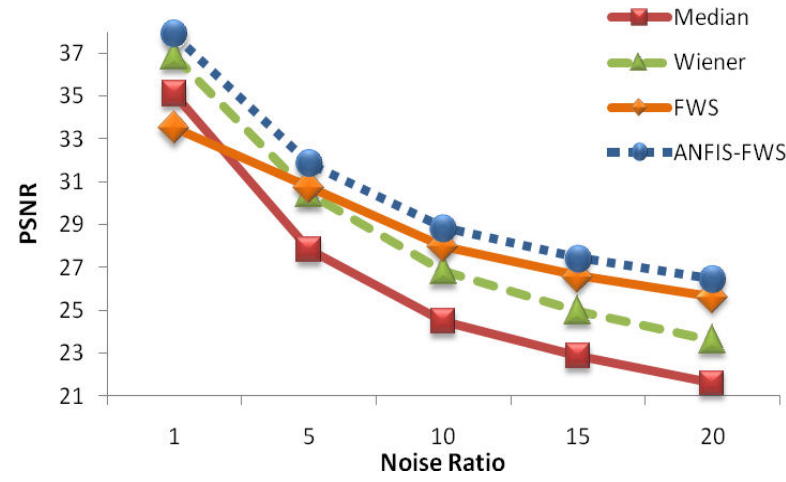

Figure 10. Comparison of proposed method performance and other methods by MSE

Furthermore, for more comparison, MSE and PSNR results from the ANFIS-FWS preprocessing method and obtained results from different methods, such as Wiener, FWS and Median filter is compared and evaluated. As figure 9 and figure 10 can be seen the proposed method has better accuracy and efficiency than mentioned methods.
In addition to statistical experiments, visual experiment results analyzed in this study. As a sample of USTB [31] database in figure 11,12 for $1 \%, 5 \%$ respectively can be seen that the preprocessing ANFIS-FWS how removes Gaussian noise effectively. After obtaining denoisy image, verification system applies on image and the accuracy results on noisy image and denoisy image is calculated and evaluated. Experimental results for the proposed method are shown in table 3 and compared this same method without denoising, obtains higher recognition rate.

\section{CONCLUSION}

One of reasons in low performance of a verification system is noise on captured images that can enhance using efficient denoising method. A hybrid two stages method is proposed in this paper. In the first stage, noisy pixel is identified using ANFIS and then in the next stage only this pixel using FWSM is changed. The proposed method is very effective to reduce impulse noise for enhancement of a verification system.

In this paper various experiments done to be able to evaluate reasonably. The experiments indicate the proposed method has appropriate performance statistically and visually than fuzzy based such as Wiener, Fuzzy Wavelet Shrinkage, and Median based methods. Also Efficiency of our method is shown in several Comparison results.

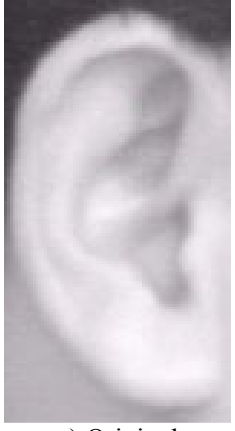

a) Original

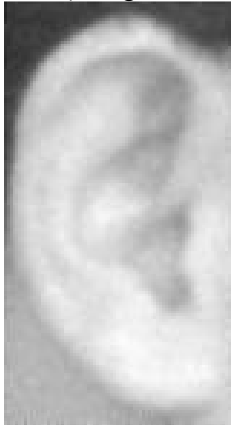

d) Wiener

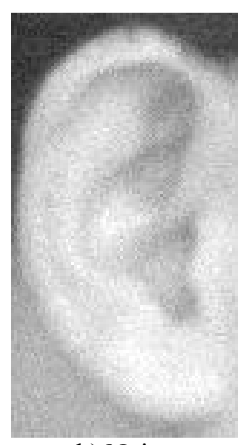

b) Noisy

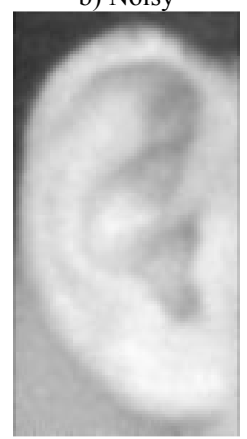

e) FWS

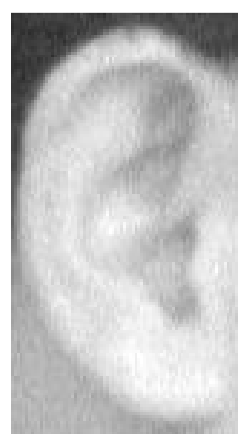

c) Median

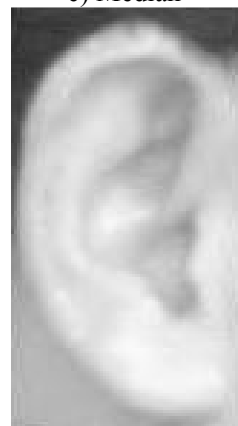

f) ANFIS-FWS
Figure 11. Comparison of the enhanced images of the methods for the ear by Gaussian noise $1 \%$. a) Original image, b) Noisy image, c) Median filter, d) Wiener filter, e) FWS, f) Proposed (ANFIS-FWS) 


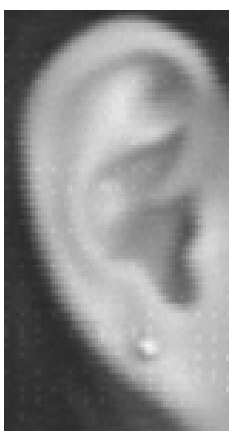

a) Original

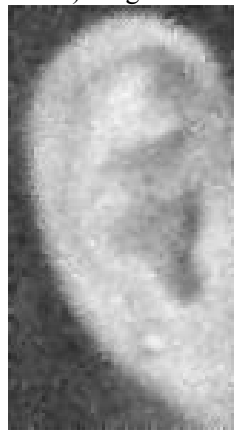

d) Wiener

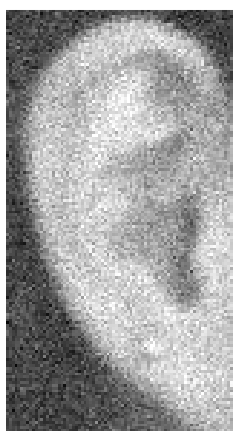

b) Noisy

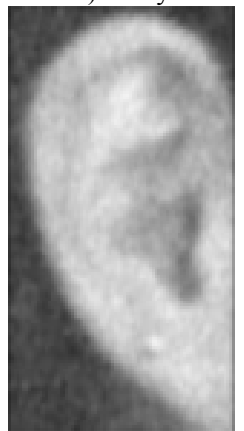

e) FWS

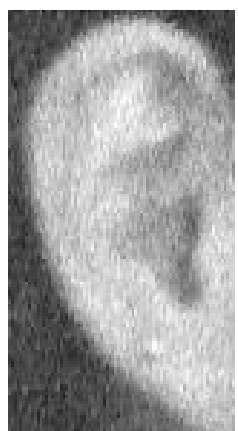

c) Median

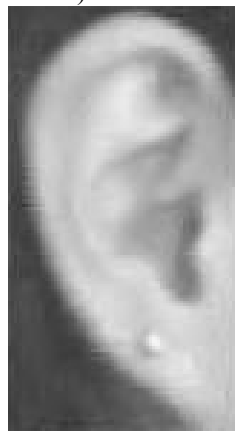

f) ANFIS-FWS
Figure 12. Comparison of the enhanced images of the methods for the ear by Gaussian noise $5 \%$. a) Original image, b) Noisy image, c) Median filter, d) Wiener filter, e) FWS, f) Proposed (ANFIS-FWS)

Table 4. Denoising algorithm accuracy for noisy and denoisy images by $10 \%$ noise.

\begin{tabular}{c|cc|cc}
\hline $\begin{array}{c}\text { Class } \\
\text { numbers }\end{array}$ & $\begin{array}{c}\text { Noisy } \\
\text { images } \\
(\mathbf{1 0} \%)\end{array}$ & $\begin{array}{c}\text { Denoisy } \\
\text { images }\end{array}$ & $\begin{array}{c}\text { Noisy } \\
\text { images } \\
(\mathbf{1 5} \%)\end{array}$ & $\begin{array}{c}\text { Denoisy } \\
\text { images }\end{array}$ \\
\hline \hline $\mathbf{5}$ & 73.3 & 90.0 & 66.6 & 90.0 \\
$\mathbf{1 0}$ & 66.6 & 86.6 & 73.3 & 80.0 \\
$\mathbf{1 5}$ & 68.8 & 83.3 & 71.1 & 81.1 \\
$\mathbf{2 0}$ & 68.3 & 83.3 & 71.6 & 81.6 \\
$\mathbf{2 5}$ & 68.0 & 82.0 & 70.6 & 82.0 \\
$\mathbf{3 0}$ & 67.7 & 78.8 & 70.0 & 81.1 \\
$\mathbf{3 5}$ & 67.6 & 79.5 & 69.5 & 79.5 \\
$\mathbf{4 0}$ & 68.3 & 79.1 & 69.5 & 80.0 \\
$\mathbf{4 5}$ & 68.1 & 78.8 & 70.0 & 78.8 \\
$\mathbf{5 0}$ & 68.0 & 79.3 & 68.8 & 78.6 \\
$\mathbf{5 5}$ & 67.8 & 79.7 & 68.6 & 78.4 \\
$\mathbf{6 0}$ & 68.3 & 78.8 & 67.7 & 78.8 \\
\hline
\end{tabular}

\section{REFERENCES}

[1] Tavassoli. S, Yaqubi. M, Rezvanian. A. 2009, "A Survey on Feature Extraction Approaches for Palm and Fingerprint", In Proceedings of the 12th Iranian Student Conference on Electrical Engineering (ISCEE 2009), Tabriz, Iran, pp. 1-6.

[2] Yaqubi. M, Mahmoudi. F, Motamed. S, Hamidi. M. 2008, "Palmprint Recognition using HMAX Model", In Proceedings of the first Iranian Conference on Data Mining (IDMC 2008), Tehran, Iran, pp. 1-5.
[3] Karim Faez, Sara Motamed, Mahboubeh Yaqubi, "Personal Verification using Ear and Palm-print Biometrics" In Proceedings of the IEEE International Conference on Systems, Man, and Cybernetics (SMC2008), Singapore, Malaysia, pp. 3727-3731, 2008.

[4] Foroughi. H, Rezvanian.A, Paziraee. A. 2008, "Robust Fall Detection Using Human Shape and Multi-class Support Vector Machine", In Proceedings of the Sixth Indian Conference on Computer Vision, Graphics \& Image Processing (ICVGIP 2008), pp. 413-420.

[5] D.B.L. Bong, R.N. Tingang, and A. Joseph. 2010, "Palm Print Verification System", Proceedings of the World Congress on Engineering (WCE 2010), London, UK, Vol. I, pp. 1-4.

[6] Nabizadeh. S, Faez. K, Tavassoli. S, Rezvanian. A, 2010. "A Novel Method for Multi-Level Image Thresholding using Particle Swarm Optimization Algorithms", In Proceedings of the 2010 2nd International Conference on Computer Engineering and Technology (ICCET 2010), Vol. 4, pp. 271-275, Chengdu, China.

[7] M. Kaur, M. Singh, A. Girdhar, Parvinder S. Sandhu, 2008,"Fingerprint Verification System using Minutiae Extraction Technique", Proceedings of the World Academy of Science, Engineering and Technology, Vol. 36, pp. 497502 .

[8] Sarkar. I, Alisherov. F, Tai-hoon Kim, and Bhattacharyya. D, 2010. "Palm Vein Authentication System: A Review", International Journal of Control and Automation, Vol. 3, No. 1, pp. 27-34.

[9] S. Cimato, M. Gamassi, V. Piuri, R. Sassi and F. Scotti, 2009. "A Multi-biometric Verification System for the Privacy Protection of Iris Templates", In Proceedings of the International Workshop on Computational Intelligence in Security for Information Systems (CISIS 2008), Advances in Soft Computing, Vol. 53, pp. 227-234.

[10] Rafael C. Gonzalez, Richard E. Woods, 2008. "Digital Image Processing", 3rd Edition, Pearson Prentice Hall.

[11] Yaqubi. M, Faez. K, Motamed. S, 2008. "Ear Recognition Using Features Inspired by Visual Cortex and Support Vector Machine Technique", In Proceedings of the IEEE International Conference on Computer and Communication Engineering (ICCCE 2008), pp. 533-537, Kuala Lumpur, Malaysia.

[12] Rezvanian. A, Faez. K, Mahmoudi. F, 2008. “A Two-Pass Method to Impulse Noise Reduction from Digital Images based on Neural Networks", Proceedings of the International Conference on Electrical and Computer Engineering (ICECE 2008), pp. 400-405, Dhaka, Bangladesh.

[13] Rezvanian. A, Rezvanian. S, Khotanlou. H, 2009. "A New Method to Impulse Noise Reduction from Medical Images Using Cellular Automata", In Proceedings of the 17th Iranian Conference on Electrical Engineering (ICEE 2009), Vol. 8, pp. 53-58, Tehran, Iran.

[14] Tavassoli. S, Rezvanian. A, Ebadzadeh. M.M, 2010. "A New Method for Impulse Noise Reduction from Digital 
Images Based on Adaptive Neuro-Fuzzy System and Fuzzy Wavelet Shrinkage", In Proceedings of the 2nd International Conference on Computer Engineering and Technology (ICCET 2010), Vol. 4, pp. 297-301, Chengdu, China.

[15] Tavassoli. S, Yaqubi. M, Rezvanian.A, Ebadzadeh. M.M, 2010. "Enhancement of Ear Verification System Performance Using a New Hybrid Denoising Approach (ANFIS-FWS)", In Proceedings of the 2010 First International Conference on Integrated Intelligent Computing, pp. 200-204, Bangalore, India.

[16] Rezvanian. A, Faez. K, 2009. “A Hybrid Method for Impulse Noise Removal from Digital Images Using Artificial Neural Network and Cellular Automata", In Proceedings of the 14th International CSI Computer Conference (CSICC 2009), pp. 1-4, Tehran, Iran.

[17] Li Wang, Ji Zhu, 2010. "Image Denoising via Solution Paths", Annals of Operations Research, Vol. 174, No. 1, pp.3-17.

[18] Shim. S, Malik. A, Choi. T, 2010. "Pre-Processing for Noise Reduction in Depth Estimation", Proceedings of SPIE, Vol. 7546, pp. 754625-75469.

[19] Bigand. A, Colot. O, 2010. "Fuzzy Filter based on IntervalValued Fuzzy Sets for Image Filtering", Fuzzy Sets and Systems, Vol. 161, No. 1, pp. 96-117.

[20] Saeedi.J, Moradi M. H, Faez. K, 2010. “A New Waveletbased Fuzzy Single and Multi-Channel Image Denoising, Image and Vision Computing", Vol. 28, pp. 1611-1623.

[21] Zhang. D, Nishimura. T, 2010. "Pulse Coupled Neural Network based Anisotropic Diffusion Method for 1/f Noise Reduction, Mathematical and Computer Modelling", Vol. 52, pp. 2085-2096.

[22] Camarena. J, Gregori. V, Morillas. S, Sapena. A, 2010. "Two-Step Fuzzy Logic-based Method for Impulse Noise Detection in Colour Images", Pattern Recognition Letters, Vol. 31, pp. 1842-1849.
[23] Chai. J, Ying. J, Li Li, 2010. “A Fuzzy Video Pre-Filtering Method for Impulse Noise Reduction", In Proceedings of the International Conference on Test and Measurement (ICTM 2009), Vol. 1, pp. 176-183, Hong Kong.

[24] Pei-Yin Chen, Chih-Yuan Lien, Yi-Ming Lin, 2008. "A Real-time Image Denoising Chip", In Proceedings of the IEEE International Symposium on Circuits and Systems (ISCAS 2008), pp. 3390-3393, Seattle, USA.

[25] Chena. H, Wanga. W, 2009. "Efficient Impulse Noise Reduction via Local Directional Gradients and Fuzzy Logic", Fuzzy Sets and Systems, Vol. 160, Pp. 1841-1857.

[26] Kang. CH, Wen-June Wang, 2009. "Fuzzy Reasoningbased Directional Median Filter Design", Signal Processing, Vol. 89, No. 3, pp. 344-351.

[27] Rezvanian. A, Ebadzadeh. M. M, Rezvanian.S, 2008. "An Efficient Method for Impulse Noise Reduction from Digital Images Using Artificial Neural Networks", In Proceedings of the 2nd Joint Congress on Fuzzy and Intelligent Systems (ISFS 2008), Tehran, Iran, pp. 1-8.

[28] E.J. Balster, Y.F. Zheng, R.L. Ewing, 2005.’Feature-based wavelet shrinkage algorithm for image Denoising", IEEE Transactions on Image Processing, Vol. 14, No. 12, Pp. 2024-2039.

[29] Zhou. Wang, Zhang. D, 1999. "Progressive Switching Median Filter For The Removal Of Impulse Noise From Highly Corrupted Images", IEEE Transaction on Circuit and Systems-II, Vol. 46, No. 1, pp. 78-80.

[30] Russo. F, Ramponi. G, 1996. "A Fuzzy Filter For Images Corrupted By Impulse Noise", IEEE Signal Processing Letters, Vol. 3, No. 6, pp. 168-170.

[31] Biometrics Research Centre (BRC), Available: http://www.ustb.edu.cn/resb/

[32] Schulte. S, Huysmans. B, Pižurica. A, Etienne E. Kerre, Philips. W, 2006. "A New Fuzzy-Based Wavelet Shrinkage Image Denoising Technique", Lecture Notes in Computer Science in Advanced Concepts for Intelligent Vision Systems, Vol. 4179, pp. 12-23. 